\section{P221 SAFETY AND EFFICACY OF PROCEDURAL SEDATION AND ANALGESIA IN PEDIATRIC ONCOLOGICAL PATIENTS}

Saba Laila Aslam*, Anwar-ul Haque, Muhammad Tariq Jamil. The Indus Hospital, Karahi, Pakistan

\subsection{6/archdischild-2019-epa.571}

Background and Aim Procedural sedation and analgesia is the standard of care for painful procedures in children that require immobility. Children with cancer are subjected to many procedures for their treatment which are painful and cause anxiety in them. Our aim was to assess the safety and efficacy of procedural sedation and analgesia in pediatric oncological patients in a large tertiary care hospital in Karachi.

Methods A retrospective study was done and records were reviewed of children receiving PSA (procedural sedation and analgesia) for pediatric oncological procedures. This included patients for oncology procedures (lumber puncture, intrathecal chemotherapy and/or bone marrow aspiration \pm trephine). PSA was provided by non-anesthesiologists. These patients were assessed according to PSA protocol guidelines by American Society of Anesthesiology (ASA). Low dose Ketamine (0.5 $\mathrm{mg} / \mathrm{kg}$ ) and Propofol $(2 \mathrm{mg} / \mathrm{kg})$ were used.

Results A total of 1216 oncological procedures were performed out of which lumber puncture was the commonest procedure performed $(n=956 ; 78.6 \%)$ followed by bone marrow aspirate only $(n=137,11.3 \%)$ and both $(n=123$, $10.1 \%)$. A total of 565 children were enrolled in the study out of which majority (65.1\%) were males and $34.9 \%$ were females (Table 1$)$. Only eight $(0.7 \%)$ of the patients were found to have hypoxia as an adverse effect of propofol-ketamine drug with $50 \%$ procedures utilizing propofol $1 \mathrm{mg} / \mathrm{kg}$ for sedation.

Conclusion This study concludes that the combination of Ketamine and Propofol is safe for procedures. There were no major complications. None of the patients required CPR or endotracheal intubation.

\section{P222 ANALYSIS OF MEDICAL HELP ORGANISATION FOR INTERSEX-PEOPLE IN RUSSIAN FEDERATION. THE STUDY WAS FORMED BY THE LEADING CLINICIANS AND DOCTORS OF THE ST. PETERSBURG STATE PEDIATRIC MEDICAL UNIVERSITY}

\begin{abstract}
${ }^{1}$ Anna Taits*, ${ }^{1}$ Konstantin Belozerov, ${ }^{1}$ Alexandra Guslistova, ${ }^{1}$ Kristina Omelchuk, ${ }^{2}$ Daria Alexandrova. ${ }^{1}$ The St. Petersburg State Pediatric Medical University., St. Petersburg, Russian Federation; ${ }^{2}$ intersex community representative, St. Petersburg, Russian Federation
\end{abstract}

\subsection{6/archdischild-2019-epa.572}

Backgrounds and aims Intersex-people is one of the most stigmatized social group around the world. On the example of androgen insensitivity syndrome/variation we have analyzed medical help organization in Russian Federation. Androgen insensitivity syndrome/variation is a genetically determined anomaly/variation of sex development manifested by prenatal formation typical female phenotype with
XY genotype, because of changes in the structures of androgen receptors. There are no clear clinical standards in majority countries. We used clinical cases by different Federal Districts: Saint-Petersburg, Moscow, Ural Federal District, Central Federal District, The Republic of Bashkortostan.

Results we've taken anamnesis and studied medical documentation of seven intersex-people with androgen insensitivity syndrome/variation. In $100 \%$ cases first specialist, who diagnosed AIS/V was gynecologist. The most popular complaints were amenorrhea, delayed sexual development (all cases) and inguinal hernia ( 2 of 10 cases). Then intersex-patients were diagnosed by ultrasonography, karyotyping, without sequencing and determination the level of the sex hormones FSH and LH (all cases); diagnostic laparoscopywas performed in 2 cases. Every intersex-patient was performed with gonadectomy, and after that was appointed by estrogen replacement therapy. Only 2 of 7 intersex-patients sure that their gonads were diagnosed with histologic studying. According to the words, none of the intersex-patients have been correctly and fully informed about negative reactions and changes in their organism after gonadectomy or about the risks of rejection of the operation. None of them was informed about alternatives of treatments. Nobody was consulting with psychotherapist about critical states, gender choices and hormone replacement therapy or appearance choices. But 4 intersexpeople of 7 decided to consultby themselves. So, only 2 intersex-patients of 7 use estrogen replacement therapy, but other rejected because of psychological, material or other reasons. None of intersex-patient has recommendations of consulting with other specialists in future, and because of negative past experience $50 \%$ avoid contacts with medical community. Only 3 of 7 intersex-patients control their coagulogram and 3 of 7 had fractures after rejection of hormone replacement therapy.

Conclusion we haven't found clear recommendations and clinical criteria about diagnostics, ways of treatments and social support for patients with androgen insensitivity syndrome/variation, and therefore the quality of care for intersex patients does not match for modern requirements and social challenges.

\section{P223 TO BE OR NOT TO BE? - VARICELLA ZOSTER!}

${ }^{1}$ Chaudhry Muhammad Asif Anwar*, 'Alwyn Charles, ${ }^{2}$ Emma Tierney, 'Aisling O'Riordan, ${ }^{2}$ Bart Ramsey, ${ }^{1}$ Anne-Marie Murphy. 'Department of Paediatrics University Hospital Limerick, Limerick, Ireland; 'Department of Dermatology University Hospital Limerick, Limerick, Ireland

\subsection{6/archdischild-2019-epa.573}

Background A rash can create a difficult diagnostic dilemma for the General Paediatrician.

Aim/Method Our aim is to describe the clinical presentation with clinical photos, diagnostic pathway and result of microbiological investigations in a school age child who presented to our Paediatric Emergency department (PED) with an unusual rash ultimately proven to be Varicella Zoster despite vaccination.

Results A previously well 11 years old boy presented to the PED with a rash for four days and a one day history of pyrexia, coryza and cough. The rash started behind the ears and 